\title{
Ultramicroscopy: PDF for review
}

\begin{tabular}{|l|l|}
\hline Journal & Ultramicroscopy \\
\hline \hline Article ID & ULTRAM_370 \\
\hline \hline Title & DESIGN OF A MICROFABRICATED, TWO-ELECTRODE PHASE- \\
& CONTRAST ELEMENT SUITABLE FOR ELECTRON MICROSCOPY \\
\hline \hline Version & 1 \\
\hline \hline Article type & Full-length article \\
\hline \hline Submitted & 31 May 06 \\
\hline
\end{tabular}

\section{Files submitted}

\begin{tabular}{|l||l|l||l||l|}
\hline Name & Fig No & Format & Use & Description \\
\hline \hline FinalCambie_et_al.doc & & $\begin{array}{l}\text { Manuscript } \\
\text { (Microsoft } \\
\text { Word) }\end{array}$ & & \\
& & \\
\hline
\end{tabular}




\section{DESIGN OF A MICROFABRICATED, TWO-ELECTRODE PHASE-CONTRAST ELEMENT SUITABLE FOR ELECTRON MICROSCOPY}

Rossana Cambie ${ }^{1}$, Kenneth. H. Downing ${ }^{2}$, Dieter. Typke ${ }^{2}$, Robert. M. Glaeser*2, ${ }^{2,4}$ and Jian. Jin ${ }^{1}$

1 Engineering Division, Lawrence Berkeley National Laboratory, University of California, Berkeley, CA 94720

2 Life Sciences Division, Lawrence Berkeley National Laboratory, University of California, Berkeley, CA 94720

${ }^{3}$ Physical Biosciences Division, Lawrence Berkeley National Laboratory, University of California, Berkeley, CA 94720

${ }^{4}$ Department of Molecular and Cell Biology, University of California, Berkeley, CA 94720

* Corresponding author:

363B Donner Laboratory

Department of Molecular and Cell Biology

University of California

Berkeley, CA 94720-3206

rmglaeser@lbl.gov

FAX: (510) 486-6488 


\begin{abstract}
A miniature electrostatic element has been designed to selectively apply a ninetydegree phase shift to the unscattered beam in the back focal plane of the objective lens, in order to realize Zernike-type, in-focus phase contrast in an electron microscope. The design involves a cylindrically shaped, biased-voltage electrode, which is surrounded by a concentric grounded electrode. Electrostatic calculations have been used to determine that the fringing fields in the region of the scattered electron beams will cause a negligible phase shift as long as the ratio of electrode length to the transverse feature-size is greater than $5: 1$. Unlike the planar, three-electrode einzel lens originally proposed by Boersch for the same purpose, this new design does not require insulating layers to separate the biased and grounded electrodes, and it can thus be produced by a very simple microfabrication process. Scanning electron microscope images confirm that mechanically robust devices with feature sizes of $\sim 1 \mu \mathrm{m}$ can be easily fabricated. Preliminary experimental images demonstrate that these devices do apply a 90-degree phase shift between the scattered and unscattered electrons, as expected.
\end{abstract}




\section{INTRODUCTION}

Powerful though modern electron microscopes have become, they still remain relatively poor instruments for imaging thin, unstained biological specimens. Such specimens are weakly-scattering "phase objects", which is to say that the intensity of the transmitted electron beam remains virtually equal to that of the incident electron beam. In this case, the effect of spatial variations in specimen structure is imprinted only on the phase of the transmitted wave. As was eloquently explained by Zernike, perfect images of such objects show no contrast [1]. As a result, weak phase objects must be intentionally viewed in an out-of-focus condition in order to partially convert the phase modulation into an intensity modulation.

The use of objective-lens defocus (and, at higher resolution, spherical aberration as well) is an imperfect way to produce phase contrast in images of biological macromolecules. The problem with that "simple" approach is that such phase shifts vary continuously over the spectrum of spatial frequencies. While the contrast transfer function produced as the result of an optimal trade-off between the phase distortion due to defocus and that due to spherical aberration can approximate the desired value of -1 over a relatively broad range of spatial frequencies [2], this band of high contrast is limited to rather high-resolution features of the image. Since it is necessary to have substantial contrast at low resolution in order to see biological macromolecules, however, it is often necessary to use a much larger amount of defocus. Unfortunately, when a high enough amount of defocus is used to generate contrast on the size scale of individual macromolecules, the phase shifts produced at high resolution can be many times the desired value of $\pi / 2$. As a result, the contrast transfer function then oscillates multiple times in the region of higher spatial frequencies.

Boersch published one of the earliest suggestions on how to achieve a uniform contrast transfer function for in-focus images of weak phase objects [3]. Boersch proposed that a weak electrostatic lens of the planar, three-electrode (einzel lens) design be placed in the back focal plane of the objective lens. The focused, unscattered beam would thus pass through the on-axis hole of the einzel lens, the voltage of which would be set to give a phase shift of $\pi / 2$ to the unscattered electrons. The scattered electrons, on the other hand, would pass to the side of the einzel lens, and thus experience no additional phase shift when the image was in focus (and in the absence of spherical aberration). The Boersch "phase plate" remained impractical to realize for decades, however, due to the engineering challenge of making the size of the einzel lens commensurate with the size of the diffraction pattern in the back focal plane. If the physical size of the einzel lens is too large, of course, then the scattered electrons that carry low- and intermediate-resolution information would be blocked, so they would not contribute to image contrast at those spatial frequencies.

Technology for the fabrication of devices with feature sizes of micrometers or less has existed for many years, but even these small sizes are too large to allow the desired part of the diffraction spectrum to pass the device when used in a strongly excited objective lens. Matsumoto and Tonomura have discussed the alternative of using a weak objective lens so that the scale of the diffraction pattern would be commensurate with the scale of the einzel lens [4]. Even if the size of the objective lens pole pieces is scaled to match the desired increase in focal length, however, the coefficient of spherical aberration, $\mathrm{C}_{\mathrm{s}}$, would increase to a value similar to the desired value of the focal length. 
The use of a weak objective lens must therefore lead to an unwanted loss in instrumental resolution.

Quite recently, however, correction of $\mathrm{C}_{\mathrm{s}}$ has become technically practical [5-8]. Thus it is timely to consider the option of combining the use of a weak objective lens, a spherical aberration corrector, and a Boersch-type phase contrast element in order to achieve uniform phase contrast for in-focus images. Schultheiss et al. have demonstrated that such a phase-contrast device can be fabricated on the size scale of a few micrometers, and that their device can be used to successfully apply a 90 degree phase shift to the unscattered electron beam relative to the scattered electrons. [9]

We now present an engineering analysis of the properties of an alternative, twoelectrode electrostatic device, which may be superior in some respects to the threeelectrode einzel lens as a phase-contrast element. This two-electrode device can be described as a "drift tube" that consists of a hollow, inner cylinder that is held at a voltage, V, down the axis of which the (focused) unscattered electrons would pass. This inner cylinder is then surrounded by a separate, grounded cylinder that shields the region where the scattered electrons would pass.

We first present the rationale that led us to consider a two-electrode design rather than the three-electrode design proposed by Boersch. We next discuss the electron optical constraints that govern the scale of the key dimensions in this design. Following that, we describe the microfabrication strategy that we have used to produce prototypes of such phase-contrast elements with feature sizes $\sim 1 \mu \mathrm{m}$ in scale. Finally we present some initial experimental results that provide a proof of concept that the drift-tube design is able to function as an in-focus phase-contrast aperture.

\section{RATIONALE FOR THE TWO-ELECTRODE DESIGN}

In an ideal phase-contrast device of the type proposed by Boersch, a phase shift of $\pi / 2$ would be applied to the unscattered beam while the scattered electrons would experience no phase shift at all. In order to approximate this ideal in a real device, the stray field from the biased electrode(s) must be kept to a minimum in the space where the scattered electrons pass.

In the "on-paper" design proposed by Boersch, the biased electrode of the einzel lens was fully enclosed by a grounded electrode. Such a device, while easily built on the macroscopic scale, is complicated to realize through microfabrication. Separately fabricated electrodes and supporting insulators would be difficult to align on the micrometer scale, of course. On the other hand, we were concerned that the use of continuous insulating layers to separate electrodes [9] (in a device that is fabricated as a single piece) might pose problems if exposed areas of the insulator become charged by stray electrons. In addition there could be complications resulting from radiation damage to the insulator when the device is hit by an intense electron beam.

An alternative approach is to use a physically separate guard electrode to shield the region in which the elastically scattered electrons must pass and use a vacuum gap to insulate the two electrodes. This is, in fact, the approach adopted in our design, which is shown schematically in Fig. 1. The adoption of two concentric (i.e. "inner" and "outer") electrodes rather than three, sequentially stacked electrodes (the design that is commonly used for an einzel lens) [10-12] frees one from the more complicated fabrication requirements of the traditional three-electrode design, especially when one considers the 
need to shield the region where the scattered electrons will go past the phase-shifting device. As is illustrated in the conceptual design shown in Fig. 1, the biased electrode and the surrounding (grounded) electrode must each be supported by narrow extensions to the outer part of the aperture.

It should be pointed out that in either design the usual function of an einzel lens, i.e. to focus the electron beam, should be so weak that it can be ignored. Instead, in our application its function is to alter the de Broglie wavelength of the unscattered electrons so that an integrated phase shift of 90 degrees is applied to the unscattered electrons relative to the scattered electrons. The shielded drift tube that is used for this purpose in our device can be considered as a special case of an einzel lens.

Intuitively, the two-electrode design should be fabricated with a relatively large aspect ratio (meaning the ratio of the height to the width of a trench) in order to contain the majority of the applied electric field within the device. The magnitude of the fringing field and its dependence on the aspect ratio are addressed quantitatively in the following section of this paper. To summarize, however, the largest phase shift experienced by the scattered electrons can be almost 30 percent of that experienced by the unscattered electrons if the aspect ratio is $5: 1$, but this phase shift becomes progressively smaller as the aspect ratio increases. Even when the aspect ratio is as small as $5: 1$, the value of the in-focus contrast transfer function (for a weak phase object) should still be 0.89 or more at all spatial frequencies, and thus even in this worst case the effect of the fringing field has relatively little impact.

\section{ELECTRON-OPTICAL CONSTRAINTS ON SCALE AND ASPECT RATIO}

The outer radius of a Boersch-type phase contrast element determines the value of the lowest spatial frequency (the reciprocal of the largest spatial periodicity), $S_{\text {low-pass }}$, that contributes to formation of an image. To be more complete, the low-frequency cutoff depends upon the focal length of the objective lens and on the electron wave length, as well as on the outer radius of the phase-contrast element. The complete expression for the lower cut-off frequency is

$$
S_{\text {low-pass }}=\frac{R_{\text {outer }}}{\lambda f}
$$

\section{Equation 1}

where $R_{\text {outer }}$ is the outer radius of the grounded electrode, $\lambda$ is the electron wavelength, and $\mathrm{f}$ is the focal length of the objective lens.

In a "prototype" design described below, we have been able to fabricate devices with an outer diameter of $8 \mu \mathrm{m}$, i.e. $R_{\text {outer }}=4 \mu \mathrm{m}$. Since our prototype design is for a 100 $\mathrm{keV}$ microscope, $\lambda$ is $0.0037 \mathrm{~nm}$. The objective lens is therefore operated with a focal length of approximately $5 \mathrm{~mm}$ in order keep $S_{\text {low-pass }}$ as low as $1 /(4.6 \mathrm{~nm})$. A focal length of $5 \mathrm{~mm}$ is about 2 times larger than is customary for state-of-the-art (high-resolution) electron microscopes, but not an unreasonably large value to start with for experiments designed to evaluate the proof-of-concept.

The diameter of the inner hole of any Boersch-type phase contrast element must not be too small, if one requires that all of the unscattered electrons should be transmitted through the device. The smallest (focused) waist of the unscattered-electron beam has a diameter equal to the product of the focal length of the objective lens and the full angular range of incident electrons. While it is true that the degree of collimation of the incident 
beam is an experimentally adjustable parameter, proportionally smaller values of the range of illumination angles can be realized only at the expense of a quadratic reduction of the beam intensity.

In our prototype design, we assume that the device will be used with the illumination adjusted to have a beam that is parallel to within $2 \times 10^{-4}$ radian, which provides a reasonable compromise between having adequate beam intensity and having a high degree of spatial coherence. Good spatial coherence is desired in order to ensure a high value of the envelope function at high resolution. The diameter of the focused, unscattered-electron beam would thus be $\sim 1 \mu \mathrm{m}$. In order to allow a margin of safety, the diameter of the inner hole in our prototype is $2 \mu \mathrm{m}$. The size of the inner hole, together with practical limitations on the width of the individual features of the device, determines the outer radius of the device, and thus the low-frequency cut off. It is not unreasonable, however, to think that a final device could be made with an outer radius of $2 \mu \mathrm{m}$, which would reduce the low-frequency cut off to $1 / 9.2 \mathrm{~nm}$, while keeping the radius of the inner hole at $1 \mu \mathrm{m}$. Further reduction of the lowest spatial frequency transmitted by the phasecontrast device would require increasing the size of the electron diffraction pattern at the plane of the device.

The main consideration that determines the aspect ratio of the device is that the phase shift applied to the scattered electrons (due to the fringing field) should be a small fraction of that applied to the unscattered electrons. The phase shift is determined by the path integral of the electrostatic potential:

$$
\delta \Phi=2 \pi \frac{1+e U / m_{0} c^{2}}{2 \lambda U\left(1+e U / 2 m_{0} c^{2}\right)} \int V(\mathbf{r}) d s,
$$

\section{Equation 2}

where

$\delta \Phi=$ the phase shift;

$\lambda=$ the electron wavelength;

$\mathrm{e}=$ the electron charge

$m_{0}=$ the rest mass of the electron

$\mathrm{U}=$ the accelerating voltage of the microscope;

$\mathrm{V}(\mathbf{r})=$ the three-dimensional electrostatic potential at a vector position, $\mathbf{r}$, due to the phase-contrast device;

and the integral is over the path, $\mathrm{s}$, followed by electrons as they go through the device.

The phase shift for electrons passing through the central hole of the inner (biased) electrode is easily estimated by approximating $\mathrm{V}(\mathbf{r})$ in equation 2 as a rectangular potential distribution of length equal to the length of the electrode. According to this simplified approximation, the voltage applied to the biased electrode should be about 17 $\mathrm{mV}$ for a device that is $10 \mu \mathrm{m}$ thick. In the real case, however, the voltage applied to the biased electrode will extend out into space for a considerable distance from the electrode. Although the value of the voltage outside the device will decay rapidly with distance, the extended path-integral will still make a significant contribution to the phase shift. As a result, the value estimated by approximating the voltage as a rectangle function is just an upper bound on the true value that should be applied in order to produce a 90 degree phase shift. 
Electrostatic calculations were performed in order to quantitatively estimate the three-dimensional voltage distribution, $\mathrm{V}(\mathbf{r})$, for a variety of representative feature-sizes. The relative magnitude of the fringing field that extends out beyond the guard electrode is needed in order to estimate the phase shift that would be applied to the scattered electrons. The ANSYS Multiphysics software package (http://www.ansys.com/) was used to carry out numerical analyses for a wide range of geometries. This package uses a finite element procedure to solve problems that are without any analytical closed-form solution due to the complexity of the geometry. The electrical potential is defined by interpolation functions (shape functions) that are associated with each element, and thus the potential is characterized by the values of the dependent variable in specified points called nodes.

The electrostatic analysis in this work was simplified by assuming axial symmetry about the central hole of the inner (biased) electrode of the device and by considering a two dimensional, longitudinal cross section of this axially symmetric, 3-D model. This simplification does not provide a description of the fringing field adjacent to the supporting structures (shown in Fig. 1), but this should be similar to that adjacent to the quasi-cylindrical electrodes. In addition, our simplification does not provide a description of the effect that the supporting structures will have on breaking the cylindrical symmetry near to the central electrodes, but this is certain to be small. All regions of vacuum were modeled by a relative permittivity (constant) of 1.0. The mesh required for the finite element analysis was created using PLANE121 elements, which are 8-node, charge-based electric elements. This type of element has one degree of freedom (the voltage) at each node. To achieve greater accuracy at critical points in the calculated electrical potential distribution, a finer mesh was created in the area of interest close to the inner electrode and around the corners of both electrodes.

A representative example of the results obtained by these electrostatic calculations is shown in Fig. 2 for a device that is $10 \mu \mathrm{m}$ thick. The left-hand edge of Fig. 2 corresponds to the central axis of the electrodes. The cylindrical wall of the inner (biased) electrode is represented by the first white bar, while the second white bar represents the cylindrical wall of the outer (grounded) electrode, or guard ring. Referring to Fig. 1, the parameters assumed for this particular calculation were $\mathrm{r}_{\mathrm{in}}=0.75 \mu \mathrm{m}, \mathrm{w}_{1}=1.5 \mu \mathrm{m}, \mathrm{g}_{1}=$ $1.5 \mu \mathrm{m}, \mathrm{w}_{2}=1.5 \mu \mathrm{m}$, and $\mathrm{r}_{\text {out }}=150 \mu \mathrm{m}$. All values of the three-dimensional potential scale, of course, with the voltage applied to the central electrode. Values of the electrostatic potential are represented in contours with a step size of 0.1 times the applied voltage, $\mathrm{V}$, except for the addition of the outer contour at a voltage of $0.05 \mathrm{~V}$. The voltage within the cylindrical hole of the biased electrode is nearly constant, as should be expected, but even so the approximation of this part of the potential by a uniform, rectangular step (as used above) is not absolutely correct.

Fig. 2 shows that relatively low values of the electrostatic potential extend beyond the edge of the guard electrode, out into the open area of the aperture. As a result of this fringing field, the electrons scattered at angles just beyond the edge of the guard electrode will experience an unwanted phase shift. Quantitative evaluation of the path integral through the fringing field shows that the amount of unwanted phase shift is greatest for rays that pass very close to the edge of the guard electrode, and that the unwanted phase shift falls by roughly a factor of 10 at the outer edge of the aperture. The maximum amount of unwanted phase shift due to the fringing field depends on the device thickness, of course. For a device thickness of $5 \mu \mathrm{m}$ it is less than 0.3 times the phase shift applied 
to the unscattered electrons, for a device thickness of $10 \mu \mathrm{m}$ this factor is less than 0.2 and for a device thickness of $20 \mu \mathrm{m}$ it is less than 0.12 . Thus if the CTF is set to be 1.0 at high spatial frequencies (i.e. corresponding to a phase shift of 90 degrees), it will be less than 1.0 at low spatial frequencies, and if it is set to be equal to 1.0 at low spatial frequencies, then it will be less than 1.0 at high spatial frequencies. Deviations from a perfectly flat CTF value of 1.0 will nevertheless be relatively small, even for the "worst case" of a device thickness equal to $5 \mu \mathrm{m}$, in which case one might expect the lowest value of the CTF to be $\sin \{(90-0.3 \times 90)$ degrees $)\}=0.89$.

Finally, although the results are not shown here, calculation of the strength of the electric field shows that it will be far below what would cause dielectric breakdown at even the sharpest edges of the device, when the voltage on the biased electrode is sufficient to produce a 90 degree phase shift for the unscattered electrons.

\section{MICROFABRICATION PROTOCOL}

The Phase Plate Device microfabrication requires three photolithographic masks. Two masks (Front-Electrodes and Front-Traces) are used to define the structural and electrical elements of the device on the device layer of a Silicon on Insulator (SOI) wafer. The Front-Electrodes mask contains the design of the central electrodes of the phase plate, including the guard ring. The Front-Traces mask defines the electrical elements necessary to connect the device to the electrical feed-through of the objective aperture holder. The third, Backside mask provides a large window on the backside of the SOI to release the top structure and allow the electron beam to pass through the modified objective aperture.

The wafers used to develop our microfabrication protocol were double side polished, SOI wafers with a device-layer that varied from a thickness of either $5 \mu \mathrm{m}$ or 10 $\mu \mathrm{m}$, depending upon the desired aspect ratio to be tested, and a $2 \mu \mathrm{m}$ thick buried-oxide layer located below the device layer. The silicon comprising the device layer is heavily doped (P/Boron) to decrease the resistivity $(<0.0065 \mathrm{Ohm}-\mathrm{cm})$. The handle wafers were $485 \mu \mathrm{m}$ thick and had a resistivity of $1-10 \mathrm{Ohm}-\mathrm{cm}$. Wafers were purchased from Ultrasil Corporation (Hayward, CA). Figure 3 shows schematic cross-sections to illustrate the progress made at various steps of the process flow. The main fabrication steps are briefly described as follows.

The first step is to clean the wafer and then deposit an oxide layer that serves as a mask during the high aspect ratio etch of the device layer. A pre-furnace clean was performed to remove organic residues and complex heavy metal ions. This cleaning step was done by immersing the substrate in a piranha bath $\left(21\right.$ of $\mathrm{H}_{2} \mathrm{SO}_{4}+100 \mathrm{ml}$ of $\left.\mathrm{H}_{2} \mathrm{O}_{2}\right)$ heated at $120^{\circ} \mathrm{C}$, followed by a de-ionized (DI) water rinse. A spin rinse/dry for approximately $2 \mathrm{~min}$ at $2400 \mathrm{rpm}$ followed. A $0.5 \mu \mathrm{m}$ oxide mask layer was then grown in a wet thermal oxidation process at a temperature of $1050^{\circ} \mathrm{C}$ for approximately 1 hour. The thickness of the oxide mask was selected to be sufficient to mask the front side of the wafer during the prolonged, Bosch Process that is needed to ensure that the narrow trenches become fully etched and create an additional barrier (protection) on the backside of the wafer during the Deep Reactive Ion Etch (DRIE).

The second step is to apply a photoresist layer and then expose it to the pattern on the Front-Electrodes mask. Before applying the photoresist (PR) coating, a standard dehydration bake and a hexamethyldisilazane (HMDS) treatment were performed. The 
HMDS treatment improves photoresist adhesion to the wafer surface, especially for wafers coated with oxide. A $1.2 \mu \mathrm{m}$ layer of OCG 825 G-line photoresist (PR) was then spun onto the device layer and heat-treated on a hotplate set at $90^{\circ} \mathrm{C}$ for 60 seconds. To project the micro-lithographic images of the $1 \mu \mathrm{m}$ features of the Front-Electrodes mask onto the 4" SOI wafer, a GCA 6200 Wafer Stepper was used. Exposure of the PR was followed by a post-exposure bake at $120^{\circ} \mathrm{C}$ for 30 seconds to improve the profile of the PR sidewalls. After developing the resist with OCG 934 developer, a standard de-scum procedure was performed using an oxygen plasma at $50 \mathrm{~W}$ for approximately 1 minute to clean residual PR from any opened areas. The wafer was then ready to be UV-baked. The UV-bake process cross-links the resist molecules thus increasing the selectivity for subsequent dry-etch applications.

In the third step, the Front-Electrodes mask was etched into the oxide layer, which serves as a "hard" mask. The equipment used to accomplish this step was a Lam Plasma Etcher. The process pressure and the RF power were set to 2.8 Torr and $750 \mathrm{~W}$, respectively, for this particular plasma etch process. Intermediate cooling steps were added to improve PR selectivity.

The fourth step is to create the Front-Traces. To do this the PR was first stripped and cleaned from the front side of the wafer and a new, $1.2 \mu \mathrm{m}$ layer of OCG 825 PR was spin-coated after a standard dehydration bake and HMDS treatment. A soft-bake followed. The photolithographic step to transfer the Front-Traces pattern onto the PR layer was accomplished by using a Suss Microtec MA6 Mask Aligner contact printer. A front-front alignment was performed. Then the PR was developed and UV-baked. After the oxide plasma etch the PR was again stripped in order to prepare the wafer for the front side silicon DRIE.

Having thus created both the Front-Electrodes and Front-Traces patterns in the $0.5 \mu \mathrm{m}$ oxide mask, the fifth step is to etch these patterns into the device layer. A Surface Technology Systems (STS) Inductively Coupled Plasma (ICP) etch system was used for this step. The plasma is inductively coupled at $13.56 \mathrm{MHz}$ through a matching unit and coil assembly. For SOI wafer etching, a separate $380 \mathrm{kHz}$ generator is available to bias the platen (electrode). The Advanced Silicon Etch (ASE) step, which consists of alternating cycles of etching and protective polymer deposition in order to achieve high aspect ratios in silicon, was performed using a low bias frequency recipe until the insulating oxide layer was reached. The low bias frequency recipe helps to reduce ionic charging at the insulator layer in deep and/or high aspect ratio trenches. Consequently, the potential notching or "footing" of Si structures was obviated. In this case the low frequency bias was necessary because of the long over-etch.

The sixth step is to perform photolithography with the Backside pattern. The wafer was once again cleaned, and after a standard dehydration bake the backside was spin-coated with approximately $10 \mu \mathrm{m}$ of SPR-220 (thick PR). A soft-bake followed at $90^{\circ} \mathrm{C}$ for 8 minutes. A front-back alignment was then performed using the Suss MA6 contact printer. After the exposure and before the post-exposure bake it was important to wait at least 30 minutes so that the photoactive compound in the resist was fully broken down. To improve the PR profile a soft bake at $70^{\circ} \mathrm{C}$ for $2 \mathrm{~min}$, followed by another heat treatment at $90^{\circ} \mathrm{C}$ for 4 min, was done on hot-plates. Developing the SPR 220 resist was accomplished by immersing the wafer in a solution of LDD $26 \mathrm{~W}$ developer for $3 \mathrm{~min}$. 
The thick resist was then baked in order to provide a good mask for the silicon oxide plasma etch, and for the subsequent and relatively long back-side DRIE processing.

At this point, the wafer was prepared for the Backside plasma etches. A handle wafer was used to protect the DRIE equipment's electrostatic chuck from damage when etch breakthrough occurred. The handle wafer was bonded to the front side of the device wafer using a $2 \mu \mathrm{m}$ thick photoresist layer as a bonding agent. The first etch allowed the Backside mask to be transferred onto the oxide layer. Due to the relatively large thickness of the two wafers bonded together, the gap between the top and bottom electrode of the Lam etcher was increased to $4.3 \mathrm{~mm}$. The Backside etch process consisted of two different DRIE processes. The first etch was timed, and a depth of about $300 \mu \mathrm{m}$ was reached using a standard high frequency bias recipe. The second etch step was performed using a low frequency bias recipe until the insulating oxide layer was reached $(\sim 200 \mu \mathrm{m}$ deep).

The most critical steps of the Phase Plate Process were then performed: a hydrofluoric acid (HF) vapor release, a wet debonding, a 49\%HF clean, a de-ionized (DI) water rinse and a Critical Point Dryer (CPD) dry. Following the CPD step, the devices were stripped of any residual polymers in a $300 \mathrm{~W}$ oxygen plasma. An example of one of the phase contrast devices is shown in Fig. 4.

\section{PROOF OF CONCEPT}

The results that we have been able to obtain in preliminary experiments with a first prototype of the drift-tube phase-contrast aperture demonstrate that it can be used to apply a phase shift of 90 degrees between the scattered and unscattered electron beams. The preliminary results described below were obtained with a device that was $5 \mu \mathrm{m}$ thick. The diameter of the hole in the central electrode is $2 \mu \mathrm{m}$ for this particular device, the electrode widths are $1 \mu \mathrm{m}$, and the gap between electrodes is $1 \mu \mathrm{m}$; the tip-radius of the device in this case is thus $4 \mu \mathrm{m}$.

Experiments were carried out with the device installed in a JEOL $100 \mathrm{C}$ electron microscope, operated at an electron energy of $100 \mathrm{keV}$. The top-entry stage of this microscope made it possible to use the "high contrast" specimen holder for these experiments, which meant that the focal length of the objective lens was substantially longer than it would be on a more modern, side-entry microscope.

A modified objective aperture holder was built for the microfabricated phasecontrast aperture. This device provides "coarse" mechanical positioning that allows one to move between 4 aperture locations, after which fine control of the aperture position is provided by a piezoelectric positioner (Nanocube model P-611K002, PI (Physik Instrumente) L.P., Auburn, MA). Coarse and fine controls are both provided in three orthogonal directions. The electrical circuit used to apply a voltage to the inner electrode also allowed switching to a mode in which it is possible to measure the current that is generated by secondary electrons when the electrode is hit by the incident electron beam. This feature made it relatively simple to center the phase-contrast aperture. In a further development (not currently available for the work reported here), the current generated by secondary electrons will be used as a feedback signal to drive the piezoelectric positioner, and thus maintain the centering of the aperture during use.

The specimen consisted of amorphous germanium evaporated onto a thin film of evaporated carbon. Images were recorded on a Gatan Megascan 794/20 CCD camera 
(Pleasanton, CA), and Digital Micrograph was used to provide "real-time" evaluation of the power spectra ("Thon patterns") of the images.

Figure 5 shows four examples of the power spectra of images that were recorded with different voltage values applied to the inner (drift-tube) electrode. The objective lens was intentionally defocused for this series of images in order to produce bright Thon rings (loci where the wave aberration associated with defocus is already equal to oddinteger multiples of $\pi / 2$ ) at spatial frequencies higher than the low-frequency cutoff imposed by the finite size of the electrodes. The power spectra of the images also show maxima and minima at spatial frequencies corresponding to the gap between the inner and outer electrodes of the device. Single-sideband contrast transfer is also seen at spatial frequencies for which only one side of the electron diffraction pattern, but not the corresponding Friedel mates, is blocked by the electron-opaque electrodes. In practice it is intended to limit data recovery to spatial frequencies higher than the low-frequency cutoff imposed by the radius of the grounded electrode.

The shift in the radial position of the bright Thon rings that is shown in figure 5 provides unambiguous evidence for the addition of a further phase shift when a bias voltage is applied to the phase-contrast aperture [9]. As the figure demonstrates, the additional phase shift reaches a value close to 90 degrees when the voltage is $\sim 11 \mathrm{mV}$. This is close to the value of $13.7 \mathrm{mV}$ that is expected from the numerical calculations of the electrostatic potential. As was already mentioned previously, the fringing field for such a thin ( $5 \mu \mathrm{m}$ thick) device is larger than would be ideal. Further work is therefore currently under way with devices that are both $10 \mu \mathrm{m}$ and $20 \mu \mathrm{m}$ in thickness, for which we expect - on the basis of electrostatic calculations described above - the effect of the fringing field to be considerably smaller.

Evidence of a nearly uniform phase shift at all spatial frequencies is provided by the experimental results obtained when the defocus of the objective lens was set to a value close to zero. Panel A of figure 6 compares the radial power spectra (integrated over a wedge of approximately 60 degrees in order to improve the signal-to-noise ratio) when the bias voltage was $0 \mathrm{mV}$ and $9 \mathrm{mV}$, respectively. The power spectrum obtained with zero bias is approximately that of electron shot noise. Since the spectrum of shot noise must be "white" (constant at all spatial frequencies), the fall-off in this curve provides an estimate of the modulation transfer function (MTF) of the CCD camera. The power spectrum obtained with a bias voltage of $9 \mathrm{mV}$ also falls off in a way similar to that of the MTF of the CCD camera, but starting with a signal well above that of the shotnoise background. Panel B of figure 6 then shows that when the bias voltage is returned to zero $\mathrm{mV}$ but the objective lens is defocused so as to produce the usual Thon rings, the resulting contrast oscillates with spatial frequency, and these oscillations are approximately confined to the lower bound of shot noise and the uniform phase contrast produced when the bias voltage is $9 \mathrm{mV}$.

As might be expected, the results above, demonstrating the proof-of-concept for this type of phase-contrast aperture, were obtained with some considerable experimental difficulty. Perhaps the greatest difficulty is caused by rapid contamination - and consequent charging - of the device when it is hit by the intense, focused central beam. As a result, the amount of time during which a "clean" device can be used is only about 15 - 30 minutes, and even during this period we expect that the performance of the aperture is continuously changing. It is thus fortunate that we are able to repeatedly clean 
the device by decreasing the excitation of the first condenser lens and increasing the second condenser aperture size during the cleaning operation. In addition to (in this way) greatly increasing the electron current that hits the device, the second condenser lens was adjusted so that the size of the spot hitting the tip of the electrodes was about $20 \mu \mathrm{m}$ in diameter, i.e. about twice the size of the device. Whether by heating the device sufficiently or by "sputtering" the carbon from the surface (perhaps through knock-on collisions and Auger processes), the aperture is effectively cleaned and work can again resume. The current limitations caused by contamination and charging are expected to go away, however, when design changes are implemented that will make it possible to heat the electrodes continuously during operation.

\section{DISCUSSION}

Excellent progress has been made in the development of thin carbon-film phase plates for electron microscopy over the past few years by the group of Nagayama [1316]. Earlier attempts to develop modified objective apertures in which a thin film of carbon serves as a quarter-wave phase plate, and even as a "contrast transfer function (CTF) phase" (i.e. CTF sign) corrector, were abandoned over 30 years ago [17-20], but it is now clear that the technology needed for such devices to be effective is finally at hand. Even so, the use of thin films of carbon (or other materials) as a quarter-wave plate for electrons suffers from the disadvantage that a significant fraction - about $25 \%$ or more of the signal-carrying electrons that are elastically scattered in the specimen are lost when they are either elastically scattered or inelastically scattered while passing through the quarter-wave plate. The use of a microfabricated electrostatic phase-contrast device (Boersch phase-plate), on the other hand, offers promise of being almost as convenient to use as is the carbon-film phase plate, while suffering essentially no loss of the signalcarrying electrons.

It is anticipated that the development of an electrostatic phase-contrast device must still solve two crucial problems, however. The first problem is to specify a design in which there is only a minimal electrostatic potential (fringing field) in the region of the scattered wave front, together with a process flow for fabricating that design. As we have demonstrated here, the use of concentric cylinders as the electrodes of a phase-contrast device results in a design that should produce minimal fringing fields in the path of the scattered electrons, and microfabrication of such a device is readily achieved with feature sizes in the desired range of $1 \mu \mathrm{m}$.

Electrostatic phase-contrast devices are anticipated to have one significant disadvantage relative to thin-film phase plates, however. Although both types of device must provide an open hole for the unscattered electrons that is of the same size, i.e. large enough to accommodate the full width of the angular distribution of electrons for the desired incident illumination, all electrostatic devices will block the electrons that are scattered for some interval of spatial frequencies just beyond the hole, whereas the thin film will immediately provide phase contrast at the lowest spatial frequencies outside the hole.

Attention has been appropriately given in the literature to the fact that contamination of a thin-film phase plate must be carefully avoided, and an effective way has been found to accomplish that [14]. We have not yet decided what approach to use 
for the electrostatic device shown here, but laser heating is one alternative that we are actively considering. Impact by the intense distribution of inelastically scattered electrons will contribute to heating of the central electrodes, as well. On the other hand, knock-on collisions will tend to remove carbon but not silicon at voltages below $\sim 150 \mathrm{keV}$, and thus cooling (rather than heating) of the modified aperture so as to reduce the re-supply of hydrocarbons by surface diffusion might be an effective strategy to avoid contamination. Other materials will have to be used to fabricate the electrostatic phase plate, of course, if it is desired to use it for electron energies above the threshold for knock-on damage in silicon.

Users may often find that electron microscopy is more complicated when a phasecontrast device is in place because there will be less freedom on how to control the beam size and beam intensity. This complication arises, of course, from the fact that new effects will appear when the focused, unscattered beam extends to angles larger than the central hole of the phase contrast device. Greater flexibility in varying the illumination could be achieved by the addition of a third lens in the condenser lens system. Failure to center the phase contrast device perfectly will also cause some of the lowest spatial frequencies to be represented in the image as single-sideband components rather than as Zernike-type phase contrast components. 


\section{ACKNOWLEDGEMENTS}

This work has been supported by the Lawrence Berkeley National Laboratory Director's Research and Development fund and by a CRADA between Gatan, Inc. and LBNL under contract DE-AC02-CH11231. We are pleased to acknowledge ongoing discussions of this research with members of the Soft Matter electron microscopy group at the National Center for Electron Microscopy (NCEM) at LBNL, in particular Nitash Balsara, Wen Kuo Hsieh, Christian Kisielowski, Alex Liddle, and Andy Minor. 


\section{REFERENCES}

[1] F. Zernike, Science 121 (1955) 345.

[3] H. Boersch, Z. Naturforschung 2A (1947) 615.

[2] O. Scherzer, J. Applied Physics 20 (1949) 20.

[4] T. Matsumoto, A. Tonomura, Ultramicroscopy 63 (1996) 5.

[5] P.E. Batson, N. Dellby, O.L. Krivanek, Nature 418 (2002) 617.

[6] B. Kabius, M. Haider, S. Uhlemann, E. Schwan, K. Urban, H. Rose, J. Electron Microscopy 51 (2002) S51.

[7] O.L. Krivanek, P.D. Nellist, N. Dellby, M.F. Murfitt, Z. Szilagy, Ultramicroscopy 96 (2003) 229.

[8] F. Hosokawa, T. Tomita, M. Naruse, T. Honda, P. Hartel, M. Haider, J. Electron Microscopy 52 (2003) 3.

[9] K. Schultheiss, F. Perez-Willard, B. Barton, D. Gerthsen, R.R. Schroeder, Rev. Sci. Instrum. 77 (2006) 033701.

[10] A. Adams, F.H. Read, J. Phys. E 5 (1972) 150.

[11] D.W.O. Heddle, J. Phys. E 2 (1969) 1046.

[12] O. Klemperer, M.E. Barnett, Electron Optics, $3^{\text {rd }}$ Ed. (1971) Cambridge University Press.

[13] R. Danev, K. Nagayama, Ultramicroscopy 88 (2001) 243.

[14] F. Hosokawa, R. Danev, Y. Arai, K. Nagayama, J. Electron Microscopy 54 (2005) 317.

[15] M. Tosaka, R. Danev, K. Nagayama, Macromolecules 38 (2005) 7884.

[16] K. Nagayama, Phase contrast enhancement with phase plates in electron microscopy. In: Advances in Imaging and Electron Physics, 138 (2005) 69.

[17] F. Lenz, Z. Phys. 172 (1963) 498.

[18] W. Hoppe, Acta Cryst. A26 (1970) 414.

[19] F. Thon, J. Electron Microscopy 20 (1971) 215.

[20] F. Thon, D. Willasch, Mikroskopie 28 (1973) 364. 


\section{FIGURE LEGENDS}

Figure 1. Schematic drawing that illustrates the concept of a two-electrode phase contrast device. Key features and dimensions are labeled in the figure: $\mathrm{R}_{\text {out }}$ is the outer radius of the objective lens aperture; $\mathrm{R}_{\mathrm{in}}$ is the radius of the central hole of the biased electrode; $\mathrm{L}_{\mathrm{b} 1}$ is the length of the silicon beam that comprises the biased electrode; $\mathrm{w}_{1}$ and $\mathrm{w}_{2}$ are the widths of the walls of the nearly cylindrical "biased electrode" and "grounded, guard electrode", respectively, as well as their supporting beams; and g1 is the width of the gap between the biased electrode and the guard electrode.

Figure 2. Example of electrostatic calculations performed for a representative example of the structural parameters of the phase contrast device (device thickness $=10 \mu \mathrm{m} ; \mathrm{R}_{\text {out }}=$ $150 \mu \mathrm{m} ; \mathrm{R}_{\mathrm{in}}=0.75 \mu \mathrm{m} ; \mathrm{w}_{1}, \mathrm{w}_{2}, \mathrm{w}_{\mathrm{b} 1}, \mathrm{w}_{\mathrm{b} 2}$, and $\mathrm{g}_{1}$ all $=1.5 \mu \mathrm{m}$; and $\mathrm{L}_{\mathrm{b} 1}=224.9 \mu \mathrm{m}$. These and other calculations extended to a distance of $500 \mu \mathrm{m}$ above and below the device in order to avoid artifacts from placing a grounded boundary that is unrealistically close to the device; test calculations indicated negligible changes when the grounded boundary was located $1 \mathrm{~mm}$ from the electrodes. The plane shown here is a longitudinal section through the phase-contrast element, corresponding to Section B-B in figure 1, and the central axis of the biased electrode coincides with the left hand margin in this figure. The walls of the biased electrode and the grounded electrode are shown as white bars. Since the calculation approximates the electrodes as perfect cylinders, only half of the longitudinal section is shown, and the full three-dimensional potential is effectively a figure of revolution about the axis on the left hand side of the figure

Figure 3. Schematic drawings, not to scale, of cross sections of the silicon wafer as it should appear at key stages of the microfabrication process. The schematic drawing shows only a single device on the wafer, but in reality many such devices are fabricated on a single wafer. The vertical "center line" indicates the central axis of the cylindrical, biased electrode (see figure 1 and figure 4 for additional views.) Numbered features correspond to 1 . the doped-silicon device layer; 2. the buried silicon dioxide layer of the SOI wafer; 3 . the $485 \mu \mathrm{m}$-thick silicon "handle" wafer; 4 . the $0.5 \mu \mathrm{m}$ oxide layer; 5 . the OCG 825 G-line photoresist layer; 6. the SPR 220 photoresist layer. (a) Cartoon showing the entire wafer surrounded by a $0.5 \mu \mathrm{m}$ oxide layer, which was first etched in a pattern determined by the front-electrodes mask. The front of the wafer was then coated with a $1.2 \mu \mathrm{m}$-thick layer of OCG $825 \mathrm{G}$-line photoresist, which in this panel has been used to define the pattern for the front-traces mask. The $0.5 \mu \mathrm{m}$ oxide layer was patterned in both cases by an "oxide reactive ion etch" (RIE) process. (b) Unwanted material in the device layer is then removed by a Bosch Process, for which the buried oxide layer acts as a stopping layer. (c) After a $10 \mu \mathrm{m}$ thick layer of photoresist (SPR-220) had been applied to the back side of the wafer and then exposed, selective areas of the $0.5 \mu \mathrm{m}$ oxide layer are again removed by the oxide RIE process. (d) Unwanted material in the wafer itself is then removed by the DRIE (Deep Reactive Ion Etch) process, after which all oxide, including that in the buried layer, is removed by a vapor HR release.

Figure 4. Scanning electron microscope images (recorded at $3 \mathrm{kV}$ accelerating voltage) of one of the phase contrast devices produced during the development of the process flow 
that is described in the text. (a) Low-magnification image showing the entire circular hole of the objective aperture, which has a diameter of $300 \mu \mathrm{m}$. The beam comprising the biased electrode enters from the solid piece on the left, which is insulated from the grounded, circular aperture. The guard electrode surrounding the biased electrode emerges from the inner edge of the aperture, as is also shown in figure 1. (b) Higher magnification view of the center (tip) of the phase contrast device. The long beam of the biased electrode terminates in a hollow cylinder, and this is surrounded by the nearly cylindrical guard electrode.

Figure 5. Demonstration of the increase in the relative phase of the scattered and unscattered electrons as the voltage on the biased electrode is increased. (A) Power spectrum (Thon pattern) of an image recorded with no bias voltage on the phase-contrast aperture. The departure from circular symmetry is due in the first instance to the supporting structure of the electrodes, but in addition it is believed that a minor contribution is made by imperfect centering of the unscattered beam within the hole of the biased electrode. (B) Split-screen comparison of Thon patterns obtained with and without a bias voltage of $3.6 \mathrm{mV}$. (C) Demonstration of the increased shift in the radial position of Thon rings when the bias voltage is increased to $7.2 \mathrm{mV}$. (D) The final shift in positions of Thon rings indicates that a nearly perfect 90 degree phase shift is produced by a bias voltage of $10.8 \mathrm{mV}$.

Figure 6. Demonstration of a nearly uniform, in-focus phase contrast transfer function (CTF) achieved with the drift-tube type phase-contrast aperture. Curves shown here are radial representations (integrated over an angle of 60 degrees in the 2-D power spectra) of the power spectra of images recorded for amorphous germanium evaporated onto a thin, amorphous carbon substrate. Values of the power spectra below a spatial frequency corresponding to the low-frequency cutoff imposed by the electrodes would not be used in data analysis, and thus are not shown in this figure. (A) The lower curve is the power spectrum of an in-focus image recorded with zero bias. This curve is interpreted as being the power spectrum of the electron shot-noise in the image intensity, and thus it provides an operational estimate of the modulation transfer function (MTF) of the CCD camera used to record the image. The upper curve is the power spectrum of an in-focus image recorded with a bias of $9 \mathrm{mV}$ on the inner electrode of the phase-contrast aperture. The fall off of this curve is due in part to the MTF of the CCD camera, but in addition it is likely that charging of the phase-contrast electrodes also contributes an additional envelope function that leads to a greater fall off than can be accounted for by the camera MTF. (B) The upper curve shown in panel A is shown here after subtracting the shotnoise background and correcting for the estimated MTF of the CCD camera. Superimposed on this curve is the radial power spectrum of an image recorded with zero bias but a large objective lens defocus, following the same compensation for shot noise and the camera MTF. It is apparent from this comparison that the in-focus CTF at low resolution is about 0.8 rather than 1.0 , which is partly due to the fact that the unwanted fringing field has a relatively large effect when the device thickness is only $5 \mu \mathrm{m}$, and partly due to the fact that the voltage of $9 \mathrm{mV}$ applied during this experiment was slightly less than what is needed in order to produce a phase shift of 90 degrees. 


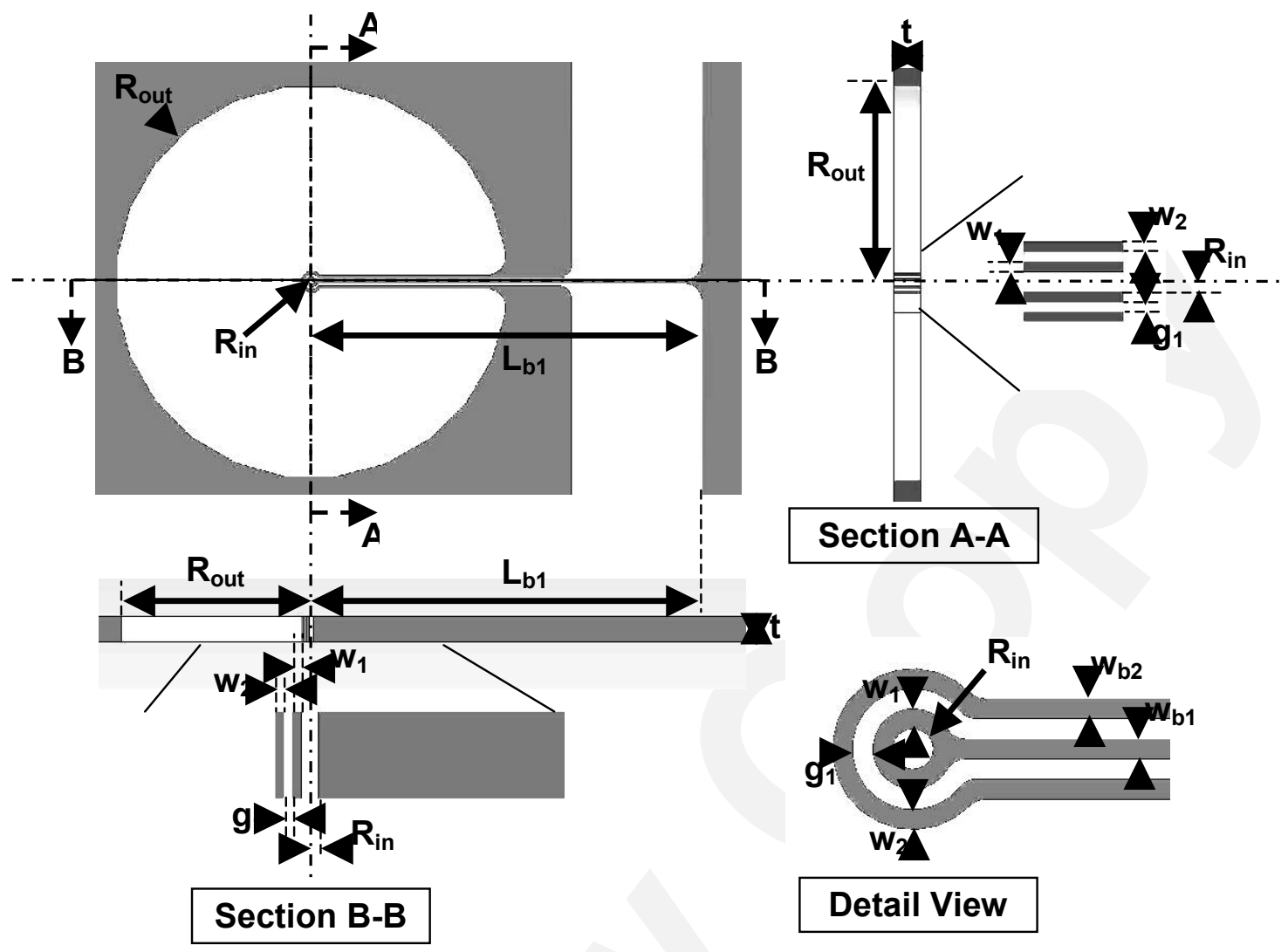

Figure 1 


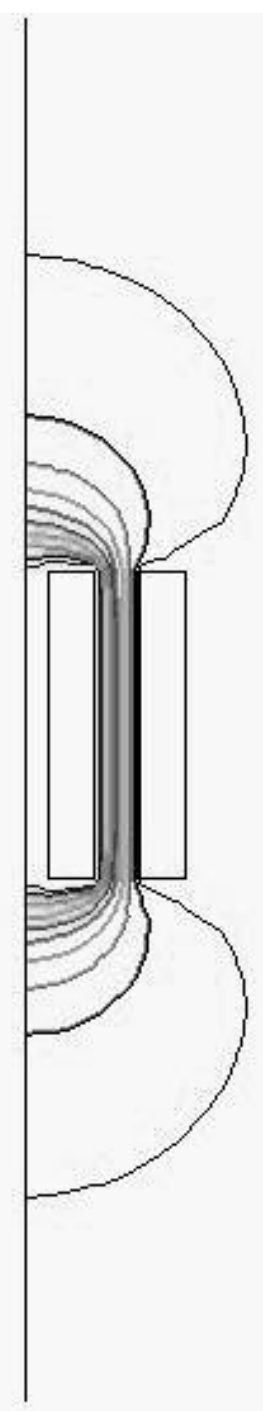

Figure 2 

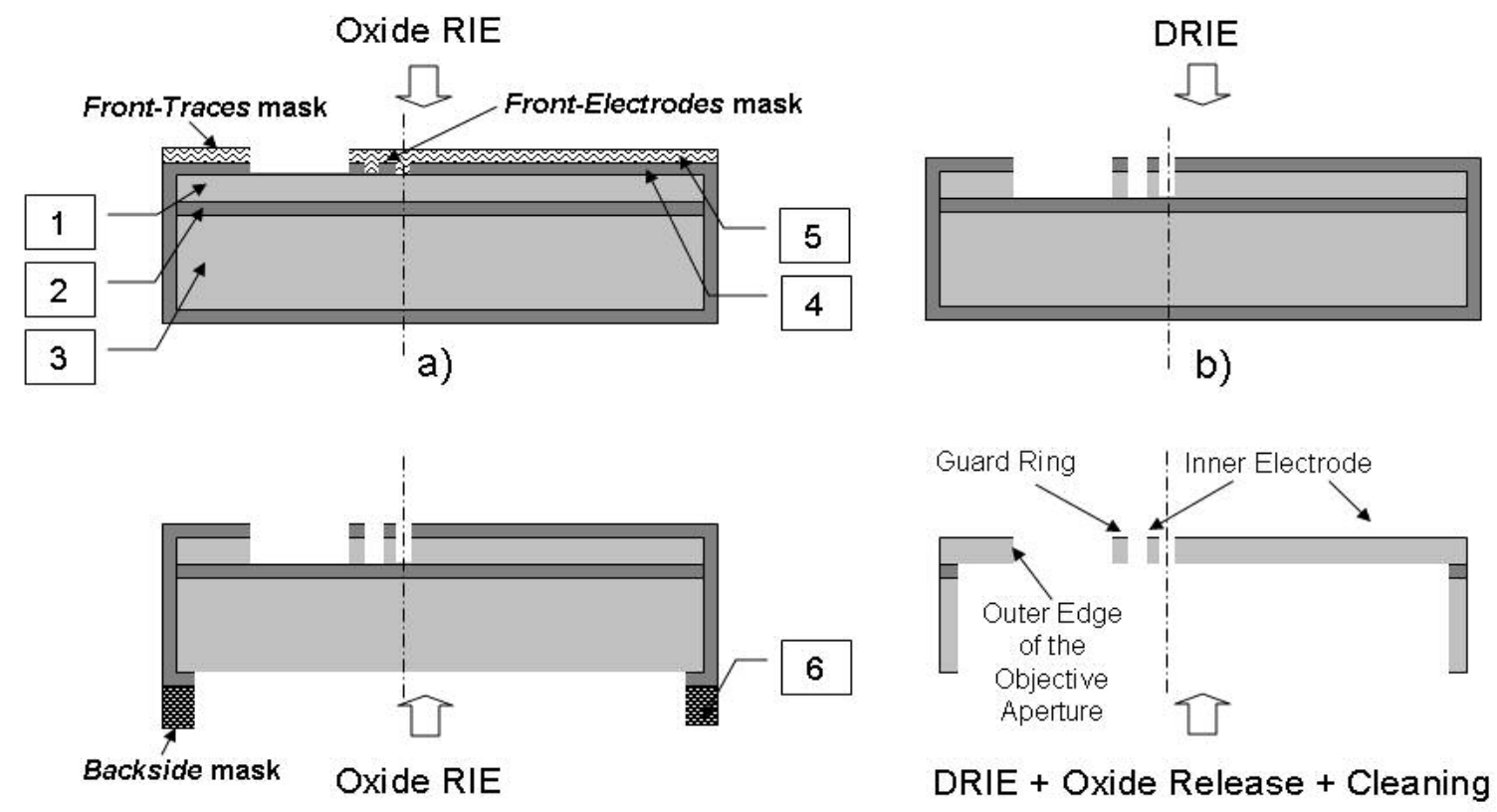

c)

DRIE + Oxide Release + Cleaning

d)

Figure 3 


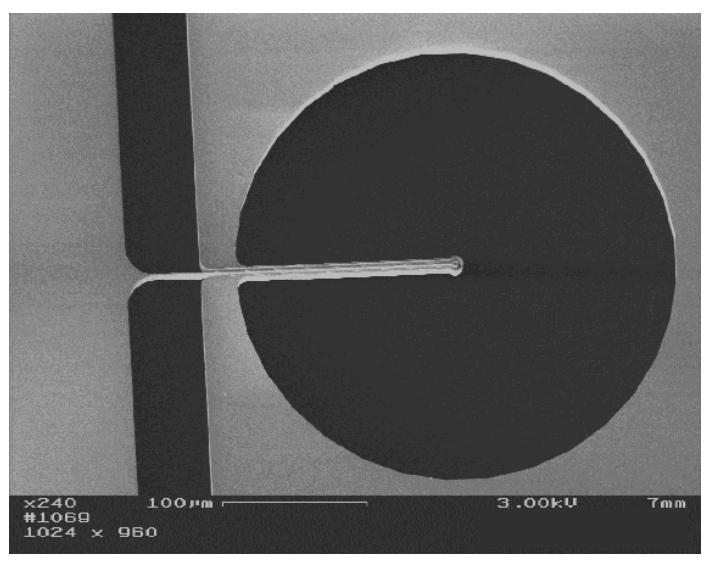

a)

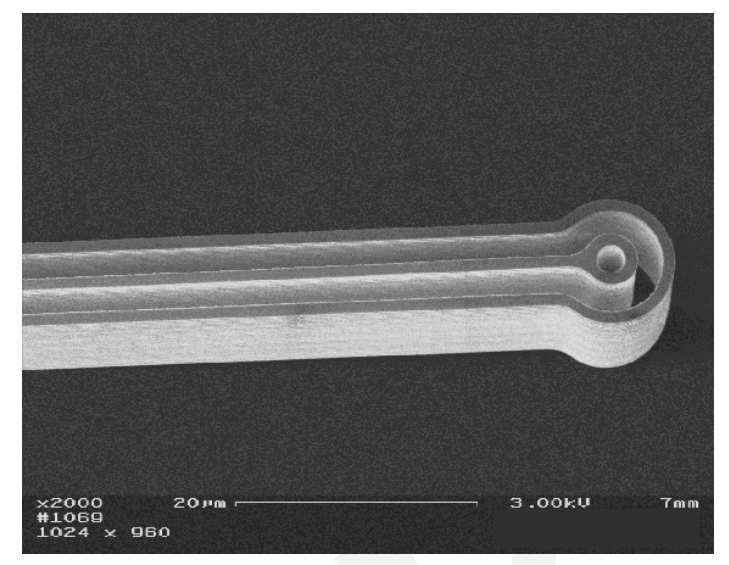

b)

Figure 4 


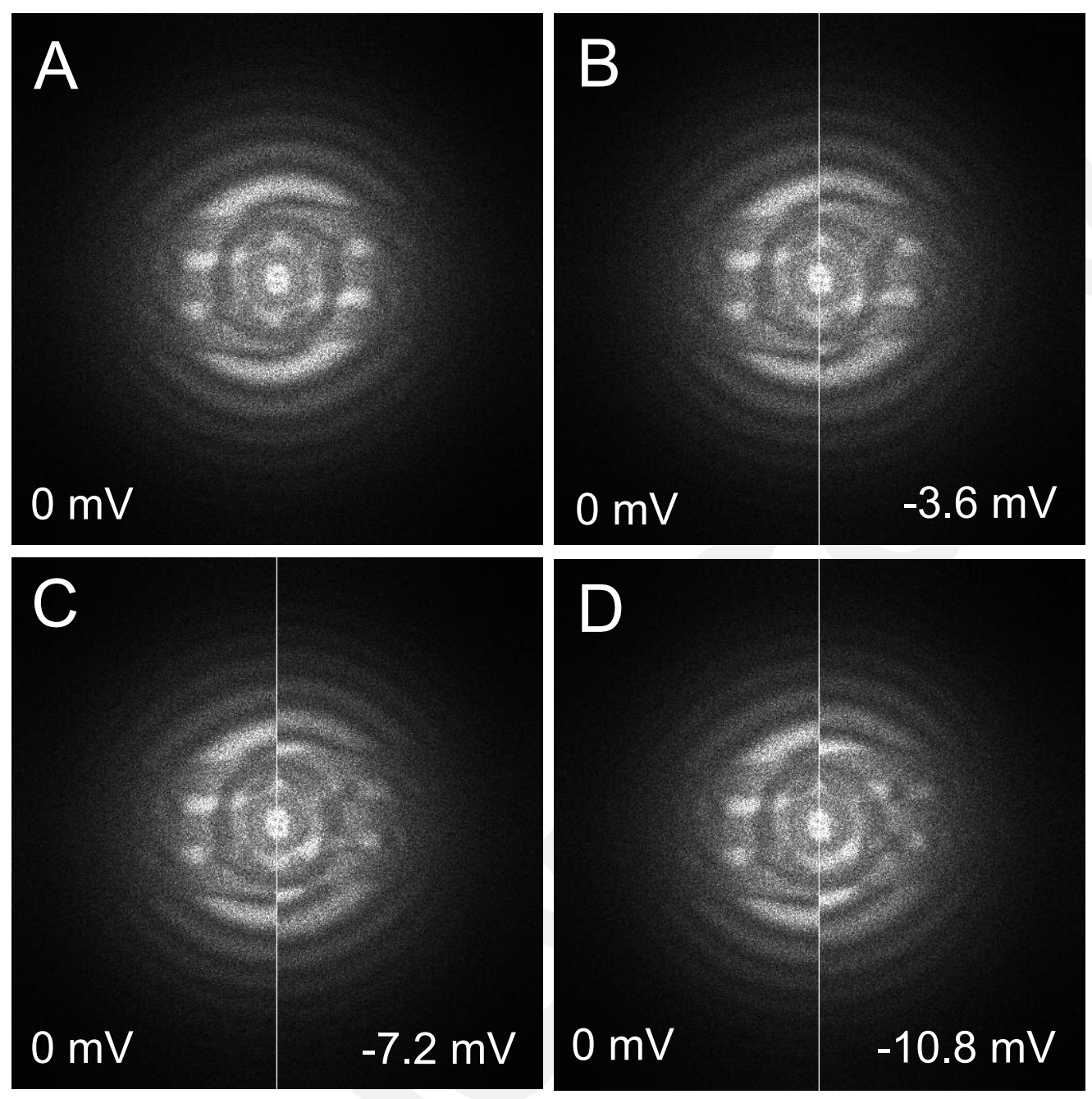

Figure 5 

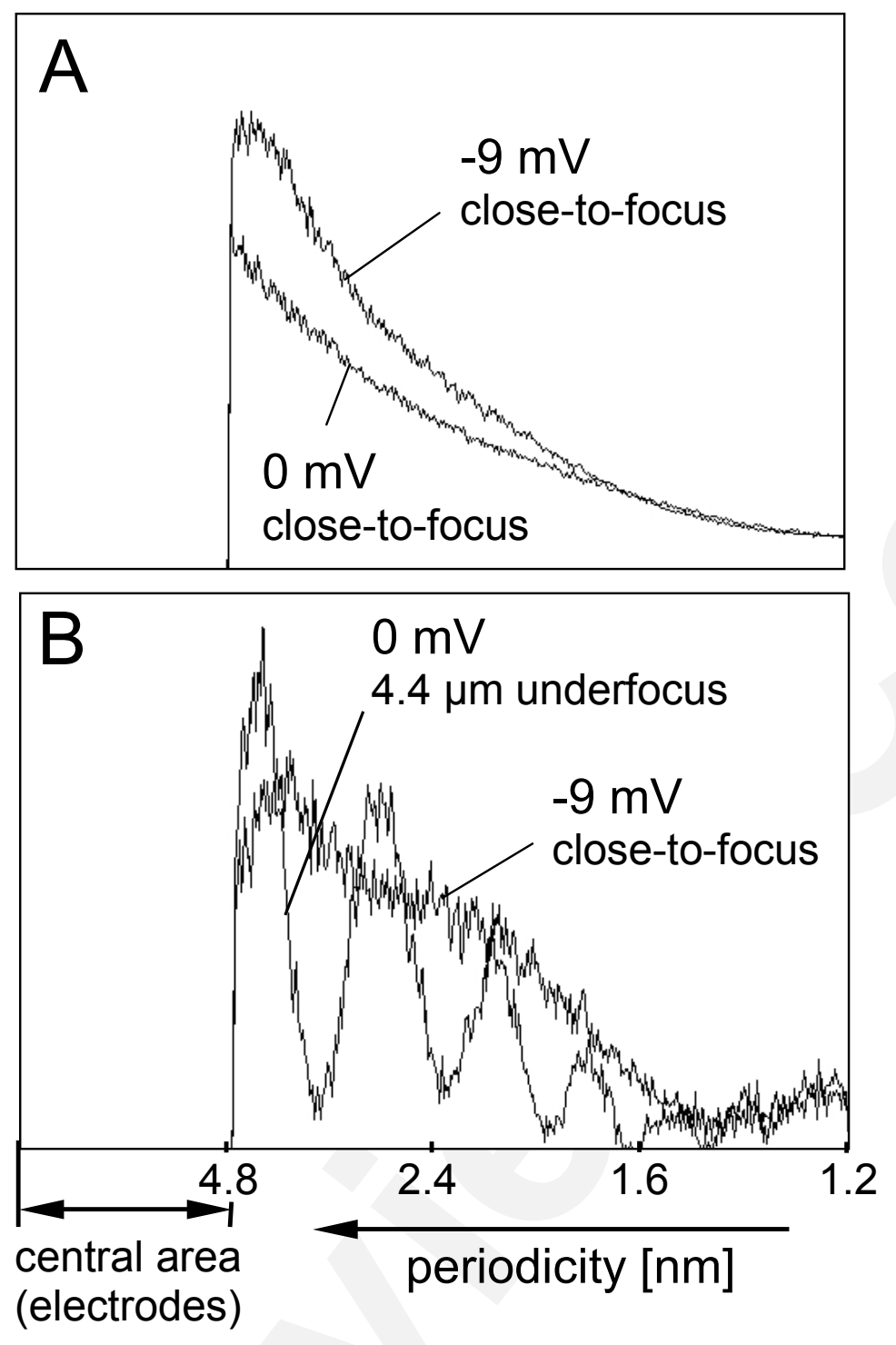

Figure 6 